\title{
DETECÇÃO DE VAZAMENTOS EM TUBULAÇÕES PREDIAIS DE GÁS ATRAVÉS DA ANÁLISE ESPECTRAL DE TRANSIENTES DE PRESSÃO
}

\author{
R. B. SANTOS ${ }^{1}$, M.M. de F. SILVA e A. M. F. FILETI \\ Universidade Estadual de Campinas, Faculdade de Engenharia Química. \\ E-mail para contato: rejanebs@yahoo.com.br
}

\begin{abstract}
RESUMO - A distribuição de gás continuamente em residências e em pontos comerciais tem se apresentado como solução de comodidade na construção civil. A confiabilidade operacional destes dutos é fundamental, sendo desejável que o surgimento de um vazamento seja identificado o mais rápido possível para minimização dos riscos associados a possíveis acidentes. O presente trabalho descreve uma técnica de detecção de vazamentos baseada na análise espectral de sinais de pressão, medidos em trechos da tubulação. Testes experimentais foram realizados em uma tubulação de cobre de $50 \mathrm{~m}$ de comprimento, em situações com e sem ocorrência de vazamentos, e em diferentes níveis de pressão. Transientes de pressão foram obtidos por oito transdutores conectados a um computador. Os testes mostraram que a análise espectral dos transientes de pressão nem sempre é eficiente para detectar vazamentos em tubulações de gás. Um segundo método de monitoramento é necessário, principalmente em situações de baixa pressão.
\end{abstract}

\section{INTRODUÇÃO}

Vazamento de gás em um sistema de tubulação é um problema sério não só do ponto de vista de segurança e ambiental, mas também perda de energia que afeta a empresa responsável pelo transporte ou distribuição do gás. Dessa forma torna-se necessário a detecção do vazamento e identificar a causa do mesmo.

Além da utilização de sistemas de tubulação como meio de transporte para gases e líquidos, estes sistemas têm sido bastante utilizados na distribuição de gás diretamente em residências e em pontos comerciais, comumente chamados de gás canalizado. O gás canalizado tem se apresentado como solução de comodidade na construção civil. Por ser transportado através de tubulações em forma contínua, o gás, combustível, não necessita de estocagem, evitando riscos com depósito e liberando área útil nas edificações. Por outro lado, há a necessidade de uma confiabilidade operacional destes dutos, minimizando os riscos de vazamentos, que podem gerar sérios problemas ambientais e financeiros. Sendo assim, é desejável que o surgimento de um vazamento qualquer em uma tubulação seja identificado e localizado o mais rápido possível para minimização dos riscos associados a possíveis acidentes. Desta forma, a detecção e localização de vazamentos se tornam os componentes mais importantes do sistema de supervisão de tubulações. 


\section{9 a 22 de outubro de 2014 \\ Florianópolis/SC}

Diante do exposto seria útil se a técnica dispusesse de um sistema e método de vazamento que pudesse determinar com exatidão a ocorrência de vazamentos de pequenas proporções em tubulações transportando gás.

Segundo Ekuakille et al. (2009) há uma forte urgência em métodos para detecção e localização de vazamentos em tubulação. Conforme os autores, a metodologia baseada no transiente de pressão pode alcançar a meta de detectar vazamentos. Os mesmos propuseram o uso da Transformada Rápida de Fourier (FFT) com o transiente de pressão para tubulações relativamente pequenas com ziguezague.

Existem vários trabalhos na literatura sobre detecção de vazamento em tubulação, por exemplo, Zhang et al. (2004), Taghvaei (2006), Toltoy et al. (2008), Yang et al. (2008), a grande parte baseada em variáveis do processo, como pressão e vazão obtidas da tubulação.

Macias (2004) descreveu uma técnica de detecção de vazamentos, em tubulações de transporte de gás, baseada na análise de transientes de pressão simulados e reais. Os transientes de pressão foram detectados e simulados para os casos de tubulações com e sem escoamento em várias condições de operação.

Souza (2002) desenvolveu uma técnica baseada na análise espectral de sinais de pressão, medidos ao longo da tubulação para favorecer a formação de ondas estacionárias com finalidade de detectar vazamentos. Os experimentos foram realizados em uma tubulação de PVC com $1250 \mathrm{~m}$ de comprimento tendo água como fluido circulante. De acordo com os resultados do autor foi possível verificar que a análise espectral dos sinais de pressão de ondas estacionárias permite detectar a presença do vazamento na tubulação com porcentagem de acerto superior a $70 \%$.

Considerando a importância de sistemas de detecção de vazamentos em redes de tubulações, o presente trabalho tem como objetivo desenvolver e testar uma técnica de detecção de vazamentos em tubulações de distribuição de gás, baseada no transiente de pressão e na análise espectral utilizando Transformada de Fourier Rápida (FFT).

\section{METODOLOGIA}

Para representação de um sistema de tubulação experimental de distribuição de gás, foi construído no laboratório da FEQ/Unicamp um protótipo experimental com a finalidade de desenvolver um sistema e método para detectar vazamentos e assim uma possível aplicação do método em uma situação real.

\subsection{Sistema experimental de aquisição de dados}

No presente trabalho, foram analisadas as características de transientes de pressão gerados pela ocorrência de vazamento de gás uma tubulação de 1/2" de diâmetro e $50 \mathrm{~m}$ de comprimento que foi montada em laboratório, com alimentação continua de gás, sob diversas condições de operação. Os vazamentos foram provocados manualmente por meio de válvulas instaladas ao longo da tubulação. A magnitude do vazamento foi controlada pela instalação de orifícios de diâmetros que variam entre 0,5 e $4 \mathrm{~mm}$.

O sistema experimental foi representado por uma tubulação de cobre de 1/2" de diâmetro, em uma estrutura de metalon (Figura 1) para simulação de um sistema de distribuição de gás em residências ou áreas comerciais. 
Para realizar os experimentos, foi utilizado ar comprimido, como fluido de trabalho, proveniente da linha que serve os laboratórios da FEQ. A pressão máxima fornecida pelo compressor responsável pelo fornecimento de ar comprimido no laboratório é de $12,3 \mathrm{kgf} / \mathrm{cm}^{2}$. A pressão máxima utilizada nos experimentos foi de $2 \mathrm{kgf} / \mathrm{cm}^{2}$.

Para monitoramento em tempo real do protótipo experimental, o sistema é composto por 8 transdutores de pressão instalados ao longo da tubulação, válvulas para simular vazamentos (variando a magnitude) e válvulas representando saídas de consumo de gás (com diâmetros de saída de 1/2”). Todos os transdutores de pressão estão interligados a um computador, onde os sinais dos mesmos são recebidos e manipulados pelo software Labview, desenvolvido pela multinacional National Instruments (NI), conforme mostra a Figura 2.

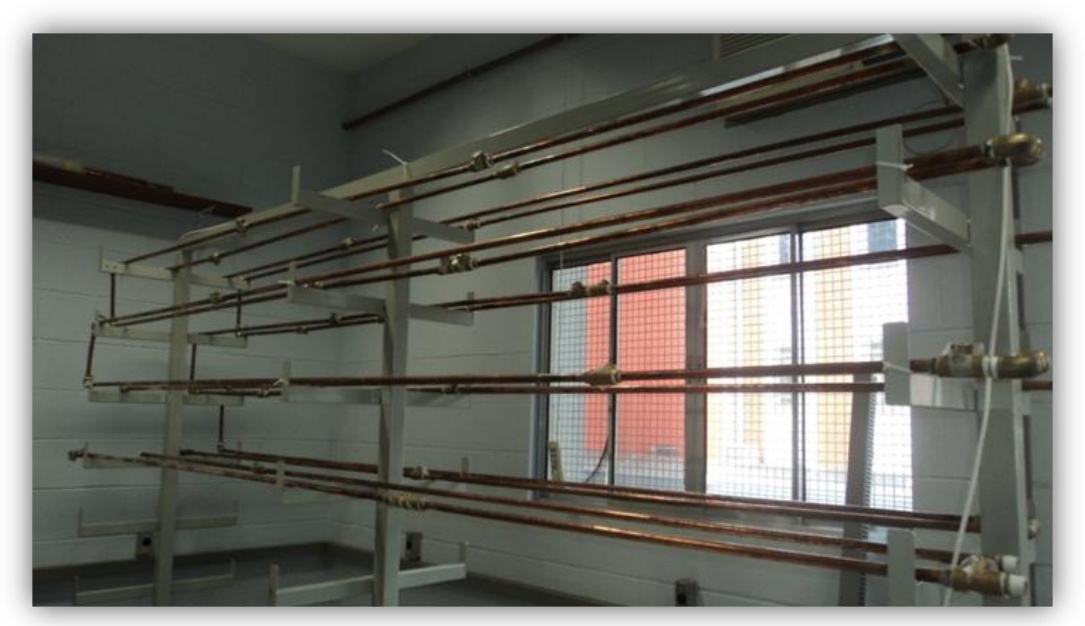

Figura 1 - Sistema experimental de tubulação para simulação de distribuição de gás em residências.

Os sinais elétrico dos transdutores de pressão, usado para medir a variação de pressão na tubulação gerada pelos vazamentos, são transmitidos a uma placa de aquisição de dados analógicodigital-analógico (ADA) da National Instruments, NI cDAQ - 9178 com 8 slots, logo em seguida, a leitura é feita pelo software LabView, com a finalidade de monitorar a planta em tempo real.

Neste trabalho, foi utilizado um filtro passa-baixa com a intenção de reduzir os ruídos presentes no sinal. O filtro passa-baixa utilizado foi o filtro ativo Butterworth de $10^{\mathrm{a}}$ ordem. O filtro ativo Butterworth foi desenvolvido no software LabView, pois esta ferramenta computacional possui um bloco para representar o mesmo.

Após os dados (sinais dos transdutores de pressão) passarem pelo filtro ativo Butterworth, sofrem a transformada rápida de Fourier que transforma o sinal analógico gerado pelo microfone em digital. A transformada rápida de Fourier associa valores numéricos às amplitudes do sinal a intervalos de tempo regulares, assim reproduzindo a forma de onda de forma discreta e não contínua. 


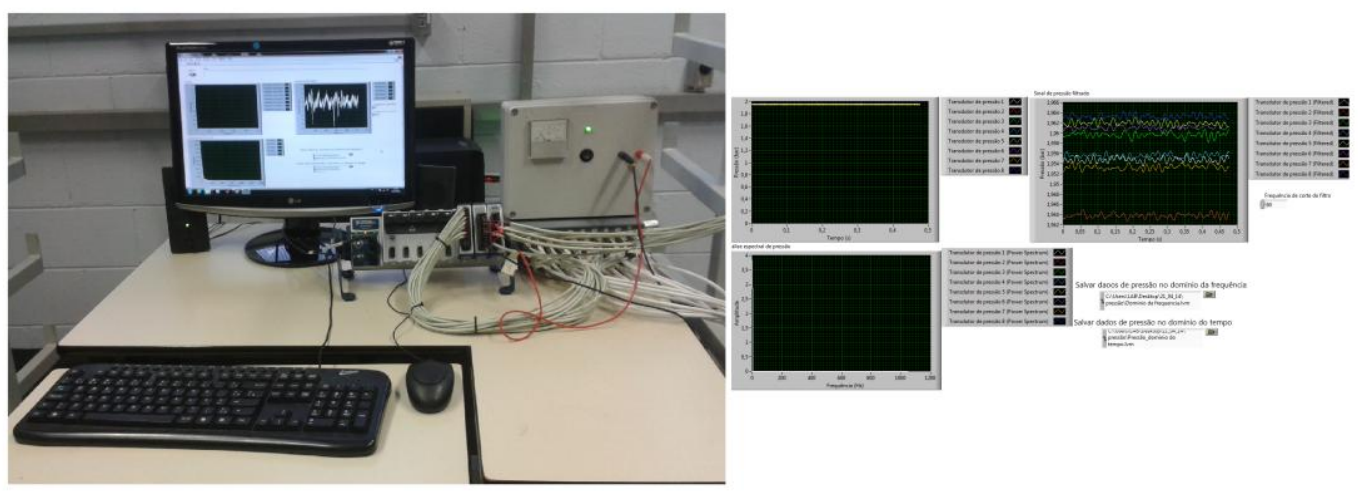

Figura 3 - Sistema de aquisição de dados utilizado no protótipo experimental de tubulação para simulação de distribuição de gás em residências utilizando o software Labview.

\subsection{Detecção de vazamentos}

O trabalho consistiu no monitoramento online da tubulação através do transiente de pressão e da análise espectral do transiente de pressão para detecção de vazamentos, com alimentação continua de ar comprimido.

Foram realizados testes experimentais em situações diferentes (conforme mostrado na Tabela 1) para analisar em tempo real o transiente de pressão ao longo da tubulação dos 8 transdutores de pressão instalados ao longo da tubulação em função do tempo.

Tabela 1 - Condições experimentais

\begin{tabular}{|l|l|}
\hline Experimentos & Situações \\
\hline 1 & Vazamentos com diâmetros entre 0,5 e $4 \mathrm{~mm}$. \\
\hline 2 & Sem vazamentos - somente ar condicionado ligado. \\
\hline 3 & $\begin{array}{l}\text { Sem vazamentos - simulação de saídas de consumo (por } \\
\text { exemplo, botijão, chuveiro, etc). }\end{array}$ \\
\hline
\end{tabular}

Com o sistema de aquisição funcionando ligado à tubulação de cobre e o software Labview fazendo aquisição de dados no domínio do tempo, foi feito a análise espectral do sinal do microfone (análise no domínio da frequência). Através da Transformada Rápida de Fourier foi possível analisar as frequências características (predominantes) em situações operacionais diferentes, com e sem vazamento. Os testes foram realizados usando diferentes tamanhos de vazamento e situações sem vazamento com o objetivo de diferenciar cada situação.

Nos testes experimentais com ocorrência de vazamento foram encontrados picos característicos de frequência no espectro através da Transformada Rápida de Fourier (FFT) aplicada ao sinal proveniente dos sensores de pressão. Por outro lado, não foram observados estes picos em situações sem vazamento. Dessa forma, percebe-se que é possível distinguir através da análise espectral, uma 
situação com ou sem vazamento.

\section{RESULTADOS}

Para sistemas de tubulação de distribuição de gás em residências ou em pontos comerciais a pressão de operação é relativamente baixa, aproximadamente $1 \mathrm{kgf} / \mathrm{cm}^{2}$. Porém, para os testes experimentais realizados neste trabalho foi $2 \mathrm{kgf} / \mathrm{cm}^{2}$.

Inicialmente foi analisado o transiente de pressão dos 8 transdutores de pressão (T) para diferentes situações, conforme Figuras 4, 5 e 6.

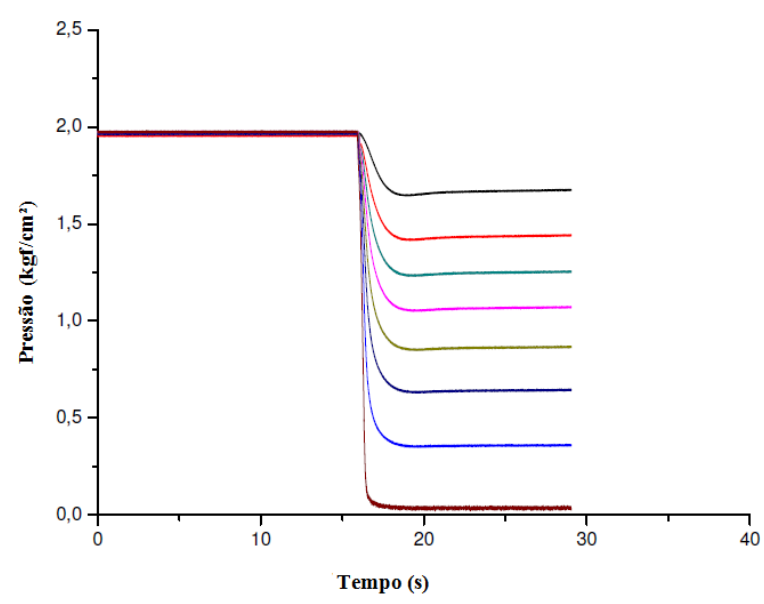

(a)

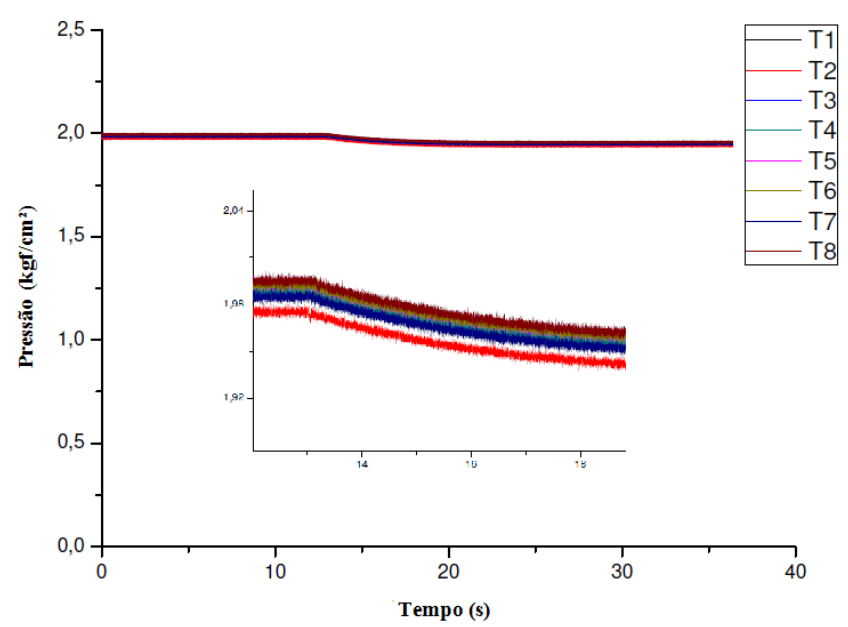

(b)

Figura 4 - Transientes de pressão com perturbações no sistema. (a) Simulação de saída de consumo com 48 metros do início da tubulação (b) Vazamento com 0,5 mm de magnitude com 1,5 metros do início da tubulação.

Percebe-se na Figura 4 que para a simulação de saída de consumo houve uma queda de pressão relativamente alta e cada sensor de pressão instalado ao longo da tubulação apresentou uma queda diferente, apontando alguma mudança no sistema (perturbação). Por outro lado, a situação com ocorrência de vazamento de magnitude $0,5 \mathrm{~mm}$ apresentou uma queda de pressão, porém foi quase imperceptível, não indicando claramente a ocorrência de vazamento.

As Figuras 5 e 6 apresentam situações com ocorrência de vazamento com tamanhos diferentes e através das mesmas percebe-se que em todas as situações houve uma queda de pressão, principalmente nos casos com tamanhos maiores de vazamentos (Figura 6) essa queda foi nítida, mostrando claramente a ocorrência de vazamento.

Através da Transformada Rápida de Fourier (FFT) foi possível analisar os espectros de frequências do transiente de pressão (análise no domínio da frequência) em situações operacionais diferentes, com e sem vazamento, conforme mostrado nas Figuras 7 e 8. 


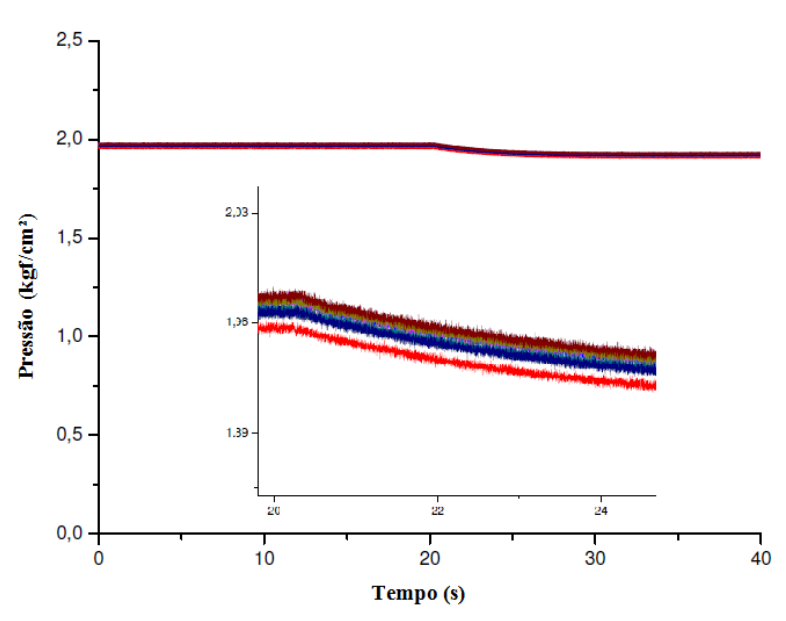

(a)

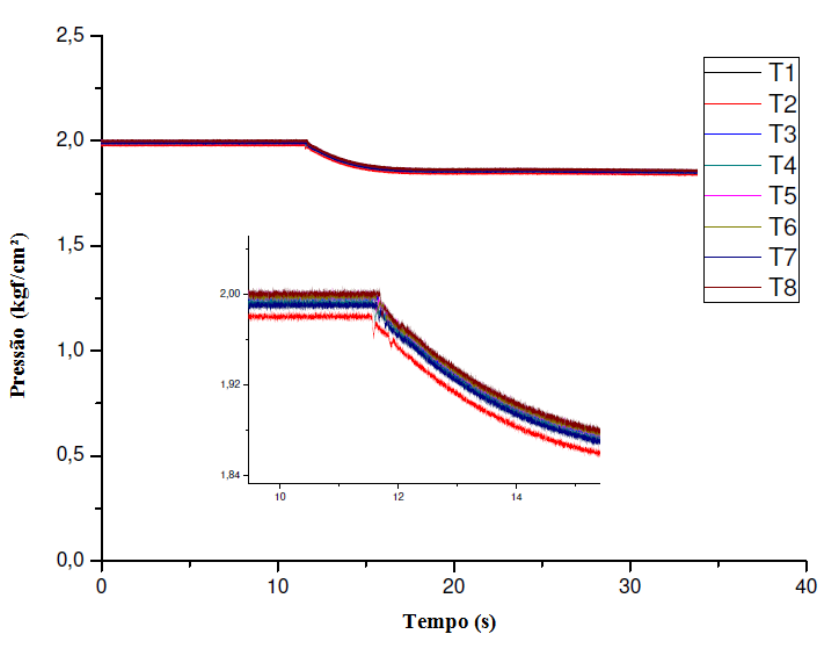

(b)

Figura 5 - Transientes de pressão com ocorrência de vazamentos. (a) Vazamento com 1,0 mm de magnitude no início da tubulação. (b) Vazamento com 2,0 mm de magnitude com 1,5 metros do início da tubulação.

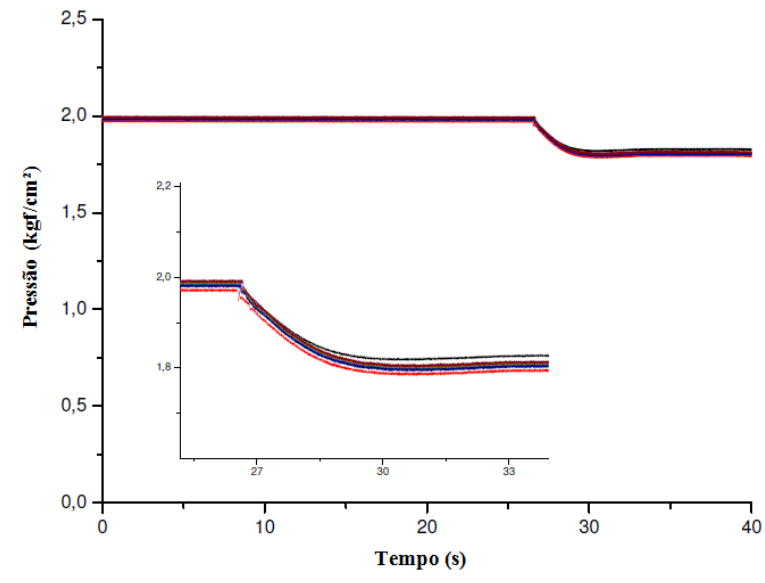

(a)

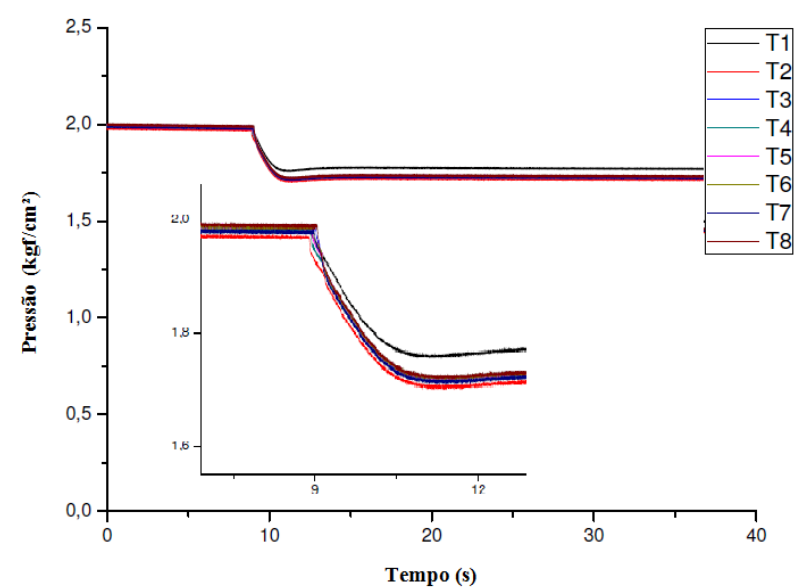

(b)

Figura 6 - Transientes de pressão com ocorrência de vazamentos. (a) Vazamento com 3,0 mm de magnitude com 1,5 metros do início da tubulação. (b) Vazamento com 4,0 mm de magnitude no início da tubulação.

Nota-se através da Figura 7 que, a situação simulando saída de consumo apresentou um espectro de potência diferente da situação sem ocorrência de vazamento, apresentando nas frequências de 0 até $20 \mathrm{~Hz}$ amplitudes mais baixas para os transdutores mais próximos da saída de consumo (próximo de $48 \mathrm{~m}$ do início da tubulação). No caso (b), sem ocorrência de vazamento, todos os transdutores apresentaram o mesmo comportamento. 

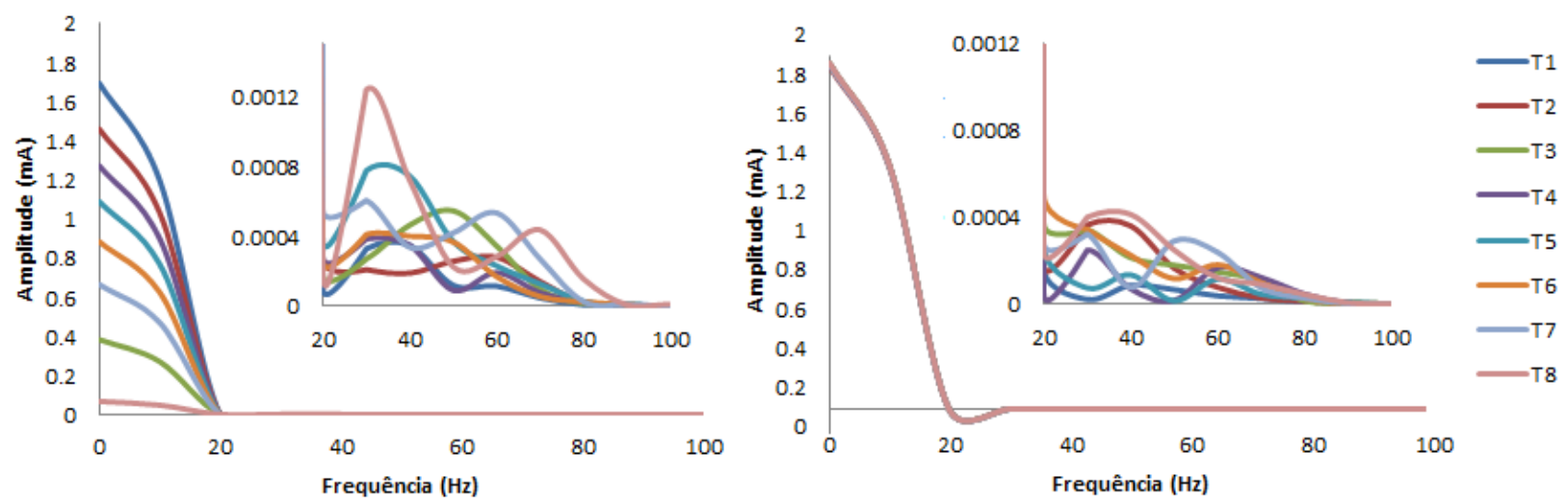

Figura 7 - Analise espectral de situações sem ocorrência de vazamento. (a) Simulação de saída de consumo com 48 metros do início da tubulação (b) Sem ocorrência de perturbação no sistema.
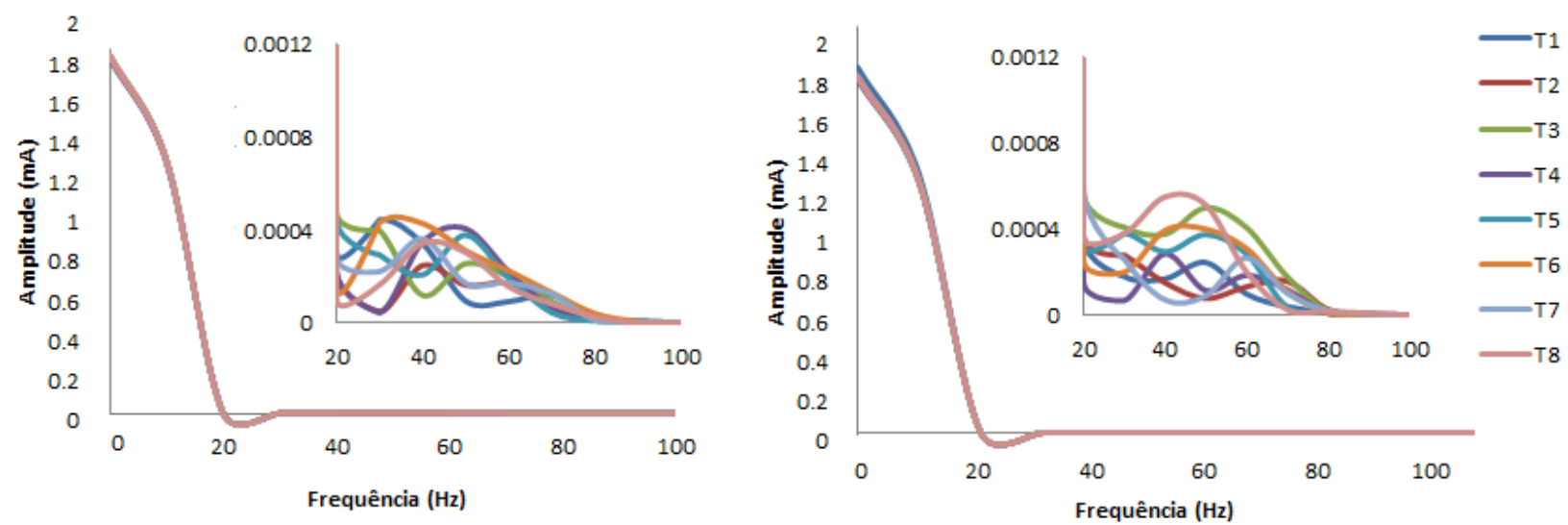

Figura 8 - Análise espectral de situações com ocorrência de vazamento. (a) Vazamento com 0,5 mm de magnitude com 1,5 m do início da tubulação (b) Vazamento com 4,0 mm de magnitude no início da tubulação.

Em todas as situações (Figuras 7 e 8) percebe-se que para todos os transdutores de pressão apresentaram picos com amplitude de aproximadamente 2 na faixa de 0 à $20 \mathrm{~Hz}$, dificultando a detecção de vazamento, exceto no caso de saída de consumo, os sensores apresentaram comportamentos diferentes (amplitudes diferentes) dependendo apenas da posição dos mesmos.

Através dos espectros de frequências (Figuras 7 e 8), levando em consideração a faixa de frequência de 20 até $100 \mathrm{~Hz}$, nota-se que para todas as situações as amplitudes apresentaram-se relativamente baixa, não diferenciando as situações distintas.

Logo, conclui-se que análise espectral do sinal de pressão é uma técnica útil para fluídos incompressíveis, como no trabalho de Souza (2002), mas não é recomendada para fluídos compressíveis, sob baixas pressões. 


\section{CONCLUSÃO}

Os resultados mostraram que através do transiente de pressão é possível verificar uma queda de pressão interna ao longo da tubulação, porém em casos com tamanhos de vazamentos menores, tornase imperceptível a queda de pressão, dificultando a detecção de vazamento.

Através da análise espectral do transiente de pressão não foi possível diferenciar situação de vazamento e situação sem ocorrência de vazamento. Pois, notou-se que todas as situações apresentaram o mesmo comportamento.

Logo, observou - se que para monitorar sistemas de tubulação de distribuição de gás é necessária outra técnica mais eficiente, pois os testes foram realizados com pressão de $2 \mathrm{kgf} / \mathrm{cm}^{2}$ e a técnica (análise espectral do sinal) se mostrou não eficiente para fluídos compressíveis e sistemas de distribuição de gás em residências e pontos comerciais funcionam com pressões mais baixas (máximo $1 \mathrm{kgf} / \mathrm{cm}^{2}$ ) dificultando a detecção de vazamento.

\section{REFERÊNCIAS}

EKUAKILlE, A. L., TROTTA, A., VENDRAMIN, G. and VANDERBEMDEM, P. FFT-Based Algorithm Improvements for Detecting Leakage in Pipelines. International Multi-Conferences on Systems, Signals and Devices, 2009.

MACIAS, I. B. Detecção de vazamentos em tubulações transportando gás. Faculdade de Engenharia Química, Universidade Estadual de Campinas, 2007. Tese (Mestrado)

SOUZA, A. L. Detecção de vazamentos em tubulações através da Análise Espectral. Faculdade de Engenharia Química, Universidade Estadual de Campinas, 2002. Tese (Doutorado)

TAGHVAEI, M.,BECK, S.B.M. and STASZEWSKI,W.J. Leak detection in pipeline networks using low-profile piezoceramic transducers. Journals Wiley Inter Science, p.1063-1082, 2006.

TOLSTOY,A., HOROSHENKOV,K.V. and BIN ALI, M.T. Detection pipe changes via acoustic matched field processing. Journal Applied Acoustics, 2009; p.695-702.

YANG, J.,WEN, Y. e LI,P. Leak acoustic detection in water distribution pipelines. World congress on intelligent control and automation, 2008; Chongqing, China, p. 25-27.

ZHANG, S., ASAKURA, T., HAYASHI, S. Gas Leakage Fault Detection of Pneumatic Pipe System Using. JSME International Journal, v. 47, n. 2, 2004. 